\title{
Correction to: Leveraging Connectivity and Automation to Improve Propulsion System Energy Sufficiency
}

Darrell Robinette ${ }^{(凶)}$, Bo Chen, Pradeep Bhat, Joe Oncken, Josh Orlando, and Neeraj Rama

\section{Correction to:}

\section{"Leveraging Connectivity and Automation to Improve Propulsion System Energy Sufficiency" in: Euroforum Deutschland GmbH (Ed.), CTI SYMPOSIUM 2018, Proceedings, https://doi.org/10.1007/978-3-662-58866-6_25}

In the original version of the book, the following belated correction has been incorporated: By mistake the two author names Pradeep Bhat and Joe Oncken were combined to one author name. This has been corrected.

The erratum chapter and the book have been updated with the change. 